\title{
Percepción docente sobre el ciberplagio académico en el marco de la enseñanza universitaria online
}

\author{
Dra. Cinta Gallent Torres ${ }^{a}$ y Dra. Isabel Tello Fons ${ }^{b}$ \\ ${ }^{a}$ Universidad Internacional de Valencia (cinta.gallent@campusviu.es) \\ b Universidad Internacional de Valencia (itello@universidadviu.com)
}

\begin{abstract}
This work will present the results of a study on cyber-plagiarism in the online teaching of Translation Studies. As Information and Comunication Technologies (ICTs) become even more integrated into people's daily life and universities' academic systems, cyber-plagiarism is on the rise. Our study was carried out by means of two tools. Firstly, we designed an ad hoc survey aimed at the faculty in the online BA in Translation and Interpretation at the Valencia International University. Secondly, a discussion group session where the same faculty was called to participate had place. The objective of these two measuring tools was to assess to which extent faculty had an accurate knowledge about cyber-plagiarism and to find out which kind of measures they use to counteract it.
\end{abstract}

Keywords: cyber-plagiarism, academic dishonesty, academic integrity, intelectual property, e-learning.

\section{Resumen}

En esta comunicación presentaremos los resultados de un estudio sobre el ciberplagio en el ámbito de la enseñanza universitaria de la traducción en la modalidad virtual; un fenómeno que se acentúa a medida que las tecnologías de la información y comunicación (TIC) se integran en la vida diaria de las personas y forman parte del sistema académico universitario. El presente estudio se ha llevado a cabo a través de dos herramientas: (i) una encuesta ad hoc dirigida al profesorado del Grado online en Traducción e Interpretación de la Universidad Internacional de Valencia, y (ii) un grupo de discusión en el que participaron estos mismos docentes. El objetivo de estas dos herramientas fue el de analizar el nivel de conocimiento de los profesores sobre el ciberplagio y las medidas que utilizan para contrarrestarlo.

Palabras clave: ciberplagio, deshonestidad académica, integridad académica, propiedad intelectual, e-learning.

\section{Introducción}

No cabe duda de que la tecnología es una puerta de acceso a una gran cantidad de información digitalizada pero, al mismo tiempo, es una fuente que genera confusión acerca 
de lo que es legal o no en el entorno electrónico (Arias Pou, 2012). Los canales por los que hoy en día se reproduce y distribuye la información o la creencia de que la utilización, en beneficio propio, de información de la red es una acción lícita al ser de acceso público, inducen al uso ilegítimo de obras cuya autoría pertenece a terceras personas.

El ciberplagio o plagio cibernético se caracteriza por la facilidad con la que la información se copia con la ayuda de los recursos disponibles en internet. Es una forma de plagio moderna que actualiza la definición clásica del término - copia de la obra (o parte de ella) de un autor atribuyéndose indebidamente la autoría (Park, 2003) -, y que resuena con fuerza en el ámbito académico. Se conoce también como deshonestidad académica y sus consecuencias pueden resumirse en que el alumno que incurre en esta práctica queda privado de unos conocimientos que le servirían en su futuro profesional. Si además recurre a ella con frecuencia, entraría en una dinámica fraudulenta a la que podría acostumbrarse, que le alejaría de cualquier objetivo formativo y, en paralelo, cuestionaría el valor de la integridad en la sociedad de la que forma parte.

De ahí la necesidad de reflexionar sobre una práctica habitual en nuestra sociedad; una práctica poco penalizada a juzgar por los casos que siguen surgiendo en relación a este fenómeno. Si históricamente el plagio se castigaba con duras penas y sanciones, ¿por qué el ciberplagio se considera un delito de menor gravedad si la única diferencia es que la información se obtiene de fuentes que se encuentran en la red? Quizá sea porque, como apuntan Sarlauskienea y Stabingis (2014), la definición de plagio o ciberplagio es diferente en los distintos países y universidades, lo que conlleva que existan varias interpretaciones sobre su significado y alcance. En cualquier caso, cabe preguntarse la razón por la que, habiendo consenso sobre la gravedad de esta práctica, siguen produciéndose estos casos en nuestras aulas.

Atendiendo a lo anterior, situamos el contexto de este análisis en la formación a distancia, modalidad en la que se imparte el Grado en Traducción e Interpretación de la Universidad Internacional de Valencia. Estos estudios están orientados a la formación de futuros profesionales de la comunicación multilingüe, capacitados para realizar traducciones e interpretaciones en distintos campos de especialidad (audiovisual, literario, jurídicoeconómico, científico-técnico, etc.). El hecho de ser una formación reglada a distancia ofrece una serie de ventajas (flexibilidad horaria, aprendizaje autónomo, facilidad de acceso desde cualquier lugar o dispositivo, metodología innovadora desde el punto de vista tecnológico, etc.) que atraen a aquellos que, hoy en día, desean cursar estudios universitarios de primer y segundo ciclo y que, por razones personales o profesionales, no pueden hacerlo en la modalidad presencial. Sin embargo, también exige que el profesorado realice un mayor seguimiento de sus estudiantes y de las tareas que entregan y, en consecuencia, que la sensibilización sobre el buen uso de la propiedad intelectual ajena se trabaje desde los primeros cursos del grado.

Dado que el e-learning conlleva una formación donde el uso de la red de redes se hace más acusado y, por tanto, la posibilidad de cometer ciberplagio puede verse incrementada con respecto a la formación presencial (Pardo Iranzo, 2014; Pavlakis and Kaitelidou, 2012), es necesario que su profesorado cuente con las herramientas y la formación adecuada no solo 
para detectarlo, sino también para disuadir al estudiantado de cometerlo. Si bien hay algunos autores que consideran que la enseñanza online favorece el plagio académico (Dickey, 2004; Underwood y Szabo, 2003), estas conductas ilícitas han existido siempre en el ámbito educativo y, por tanto, no es concluyente que a mayor distancia en el entorno de aprendizaje, mayor facilidad para cometerlas. En realidad, esta práctica no depende solo del entorno, sino del estudiante, el centro, el profesorado y la metodología empleada, factores que influyen en la adopción de estas conductas poco éticas.

Sin duda, el interés por esta práctica fraudulenta ha ido en aumento y prueba de ello son los numerosos trabajos que se publican en torno a este tema (Bautista Buenfil, Sánchez Escobedo y Canto Herrera, 2017; Comas, Sureda, Casero y Morey, 2011; Domínguez Pérez, 2015). Muchos de ellos analizan este fenómeno en el ámbito universitario y ponen de relieve el elevado índice de plagio que existe en estos niveles educativos.

El consenso general en estos estudios es que todos los esfuerzos deben ir dirigidos hacia el diagnóstico, la concienciación y la prevención de este fenómeno (Medina Díaz y Verdejo Carrión, 2016), así como hacia la reflexión metodológica y el rediseño de tareas que limiten las posibilidades del ciberplagio en el contexto académico. En este sentido, queda mucho trabajo por hacer para sensibilizar a la comunidad universitaria sobre las consecuencias que estas prácticas tienen a corto y largo plazo. Y el profesorado, aquí, tiene un papel fundamental. Por eso, nuestro interés se ha centrado en conocer su percepción acerca del alcance de este fenómeno y de las medidas y estrategias que utiliza para detectarlo y disuadirlo.

Ciertamente muchos son los estudios que analizan la visión del estudiante sobre esta práctica, pero no son tantos los trabajos que hacen hincapié en la visión de los docentes sobre el ciberplagio, sobre la responsabilidad que se les puede atribuir o las medidas que deben adoptar para contrarrestarlo (Cueva Lobelle y Ochoa Sierra, 2015; Ronda-Pérez, Seguí-Crespo, Tauste Francés, Martínez Martínez y Esteve-Faubel, 2015). Es importante, pues, realizar una revisión bibliográfica desde esta perspectiva y poner en marcha estudios que analicen su percepción como colectivo afectado para dar respuesta a cuestiones que son recurrentes en nuestros claustros; cuestiones como si debemos suspender directamente una actividad o pedir al estudiante que la rehaga si ha plagiado; o cómo podemos identificar que la conducta ha sido voluntaria o accidental; o incluso, si existe un protocolo institucional para comunicar este tipo de situaciones, etc. Sin duda, más allá del sentido común aplicable a cada caso, sería necesario contar con estudios que avalen y orienten las actuaciones de los docentes en circunstancias de naturaleza plagiaria.

En coherencia con lo anterior, este estudio pretende acercarse al problema a través de los docentes del Grado en Traducción e Interpretación de la Universidad Internacional de Valencia para conocer de primera mano su experiencia en esta materia y las medidas que adoptan para evitar que se incurra en estas prácticas. Para recabar esta información, se diseñaron dos instrumentos de medición que nos permitieron analizar el estado de la cuestión y compartir con el profesorado la percepción respecto a este fenómeno. Por una parte, se elaboró una encuesta mediante la cual identificamos el nivel de conocimiento del profesorado sobre el ciberplagio (definición, alcance, utilización de programas de detección 
antiplagio, formación recibida, factores que incitan al estudiantado a cometer estas prácticas, posibilidad de encontrar una utilidad didáctica a esta práctica, etc.); y, por otra parte, se organizó un grupo de discusión en el que participaron 16 profesores del grado (de los 29 que contestaron a la encuesta) mediante el cual se profundizó en algunas de las cuestiones ya planteadas en esta herramienta y se abrieron nuevos interrogantes que conllevarían el diseño y desarrollo de futuras acciones en esta materia. Consideramos que los resultados y conclusiones alcanzados evidencian un primer paso hacia una reflexión colectiva sobre un problema que afecta al ámbito académico, pero también a otras esferas de la sociedad.

\section{Objetivos}

El objetivo de este estudio es reflexionar sobre el ciberplagio universitario a través de su profesorado. Si bien en nuestros trabajos anteriores (Gallent Torres y Tello Fons, 2017 y 2018) hemos analizado la percepción de los estudiantes del grado de traducción sobre este mismo fenómeno, en esta ocasión nuestro interés se centra en analizar la opinión del claustro docente y tener así una visión más completa sobre el alcance de estas prácticas en nuestras aulas.

Sin ser exhaustivos, indicaremos que del objetivo principal del estudio se derivan los siguientes:

(i) Identificar el grado de conocimiento del profesorado sobre los términos plagio y ciberplagio.

(ii) Conocer si han detectado, en los últimos tres años, algún caso de plagio y en qué tipo de actividades los estudiantes lo cometieron.

(iii) Conocer cómo el profesorado detecta el plagio y cuál es su actuación posterior.

(iv) Identificar los factores que incitan al estudiantado a incurrir en estas prácticas fraudulentas.

(v) Compartir las medidas que el profesorado toma para corregir este comportamiento.

(vi) Reflexionar sobre las medidas que el centro debería implementar para sensibilizar a la comunidad universitaria sobre este fenómeno.

Más allá de reflexionar sobre estos aspectos, con este trabajo pretendemos hacer una llamada de atención a los distintos colectivos universitarios, para que, según su nivel de responsabilidad, promuevan el respeto a la propiedad intelectual ajena, fomenten el valor de la integridad académica y potencien en el alumnado la creación y desarrollo de ideas originales y propias en lugar del reciclaje y el collage de información digitalizada.

\section{Desarrollo de la innovación}

Para cumplir con los objetivos del estudio, se diseñó una encuesta semiestructurada ad hoc, titulada Encuesta sobre el ciberplagio dirigida al profesorado del Grado de Traducción e 
Interpretación, a través de la cual se recogieron las percepciones del claustro del grado que impartía docencia durante el curso académico 2018-2019. Todos los profesores $(n=29)$ contestaron a la encuesta administrada online mediante la aplicación Google Forms.

Se optó por crear un modelo de encuesta propio, adaptado a los objetivos de la investigación y a las características propias del grado; decisión que se tomó tras analizar el corpus documental existente sobre esta materia y otras herramientas creadas por otros autores para analizar el fenómeno desde esta misma perspectiva (Cueva Lobelle y Ochoa Sierra, 2015; Ronda-Pérez, Seguí-Crespo, Tauste Francés, Martínez Martínez y Esteve Faubel, 2015).

La encuesta fue validada posteriormente por cinco expertos externos de la Universidad de Valencia (UV), Universidad Jaume I (UJI) y Universidad Autónoma de Barcelona (UAB), que analizaron la viabilidad de la herramienta y propusieron las modificaciones pertinentes. Este proceso conllevó la reflexión, el análisis y la modificación de algunos ítems que redundaron en la mejora del instrumento final.

La versión resultante de la herramienta reunía un total de 27 ítems de valoración escalar, tipo Likert, con etiquetas o cuantificadores lingüísticos representados por el grado de acuerdo y desacuerdo ("totalmente en desacuerdo"/“totalmente de acuerdo") y el grado de frecuencia ("nunca"/“siempre"). También incluía ítems de respuesta dicotómica ("sí'"/"no") y otros, mayoritariamente, de respuesta abierta.

Dichos enunciados se agruparon en torno a siete dimensiones:

1) Concepto y categorización del plagio/ciberplagio (definición de los términos).

2) Experiencia previa en materia de ciberplagio.

3) Experiencia actual en materia de ciberplagio (formación recibida para su detección, conocimiento de la existencia de la normativa interna del centro en esta materia, existencia de un protocolo de actuación, etc.).

4) Factores que incitan a la comisión de ciberplagio.

5) Cuestiones metodológicas docentes (tipo de actividades planteadas, información sobre recursos (vídeos, tutoriales, enlaces, etc.) que traten este fenómeno, indicaciones sobre la elaboración de trabajos académicos, etc.).

6) Cuestions formativas; $y$

7) Medidas a adoptar para el diagnóstico, concienciación y prevención del ciberplagio (tanto por parte del centro como de su profesorado).

Además se incluyó un apartado para la contextualización del grupo en el que los participantes debían indicar sus datos personales y académicos, su experiencia docente en educación superior y, en particular,en plataformas o sistemas de gestión del aprendizaje.

Como complemento a esta herramienta se les propuso participar en un grupo de discusión principalmente por dos razones: la primera porque contestar a la encuesta implicaba invertir una cantidad de tiempo considerable (entre 30 y 45 minutos) y ampliar el número de enunciados nos parecía un tanto excesivo; y la segunda porque nos interesaba profundizar en algunas cuestiones que si se hubieran planteado a través de la encuesta, se hubieran analizado superficialmente. 
Se convocó, pues, al profesorado a una conexión por videoconferencia en el mes de octubre de 2018, a la que asistieron 16 docentes. Atendiendo a la dificultad que supone encontrar una fecha y hora que se adapte a los horarios de todos los participantes (no todos ellos residen en España), consideramos que el número de asistentes fue muy satisfactorio, ya que más de la mitad del claustro pudo participar.

Durante la sesión se debatieron cuestiones como:

- ¿Qué responsabilidad corresponde asumir al profesorado que detecta un caso de plagio? ¿Quién debe calificar y cuantificar su gravedad?

- ¿A qué se debe la disparidad de criterios docentes para gestionar cada caso?

- ¿Por qué los casos de plagio identificados suelen solucionarse de manera individual entre el profesor y el alumno y apenas trascienden a la dirección de título?

- ¿Qué cambios metodológicos debemos implementar (tanto en las clases, las actividades como en los exámenes) para evitar estas conductas?

- ¿Cómo podríamos implicar más a la institución en la lucha contra este fenómeno?

- ¿Qué tipo de apoyo espera recibir el profesorado por parte de la institución para atajar estas prácticas fraudulentas?

Poner en común ideas e inquietudes encaminadas a resolver una problemática que afecta a nuestro día a día, nos aporta, sin duda, un gran valor como profesionales.

Ciertamente, resumir en esta comunicación conclusiones, iniciativas y futuros proyectos que surgieron a raíz de este estudio es una labor harto complicada, ya que implicaría escribir un buen número de páginas sobre el alcance de este fenómeno desde la perspectiva docente. Sin embargo, sí podemos afirmar que este estudio es pionero en nuestra universidad, ya que es la primera vez que se diseña una encuesta sobre ciberplagio dirigida al claustro de un grado universitario. Su acercamiento es también distinto a otras publicaciones sobre esta materia porque combina dos instrumentos que se complementan la encuesta y el grupo de discusión-, dos herramientas interesantes desde el punto de vista de la investigación, a través de las cuales se triangula y confronta la información obtenida sobre estas prácticas; información que, sin duda, nos ayudará no solo a tener conocimiento de lo que opina nuestro profesorado sobre estas conductas, sino también a seguir trabajando, de manera coordinada, para atajar estas prácticas ilícitas.

\section{Resultados}

Para el análisis de los resultados de la encuesta, especialmente para los ítems de valoración dicotómica y escalar, el vaciado de datos se realizó con el paquete estadístico SPSS 24.0 a partir del análisis de frecuencias y porcentajes para cada enunciado; datos que se complementaron con otras mediciones estadísticas como análisis factoriales, de varianza, pruebas post hoc y comparaciones múltiples de variables. En cuanto a los ítems de respuesta abierta, estos se analizaron agrupando aquellas respuestas coincidentes e incorporando aquellas aportaciones distintas a las ya agrupadas, sistematizando así toda la información recopilada. 
Por cuestiones de espacio y de tiempo, expondremos a continuación los resultados más significativos del estudio, aquellos que responden a los objetivos principales de la investigación y que, en paralelo, abren una puerta a futuras acciones en esta materia, algunas de las cuales ya están siendo planificadas.

Empezaremos contextualizando la muestra de participantes, un total de 29 profesores del Grado en Traducción e Interpretación de la Universidad Internacional de Valencia. Todos ellos cumplimentaron la encuesta, si bien únicamente 16 participaron en el grupo de discusión. De ellos, 13\% son hombres y $87 \%$ mujeres, de 39 años de edad como media, de nacionalidad española (92\%), inglesa (4\%) y argelina (2\%), con formación académica de licenciatura/grado (10\%), máster (19\%) y doctorado $(71 \%)$, y una media de experiencia en enseñanza online de 3 años. De entre el claustro, el $31 \%$ no ha detectado casos de plagio durante los últimos tres años, mientras que el $69 \%$ sí lo ha hecho, y en más de una ocasión.

En cuanto a los resultados principales se desprende que todos ellos tiene una idea acertada sobre lo que significa plagiar o ciberplagiar, ya que coinciden, en sus definiciones, en que se trata de una "apropiación indebida e ilícita del trabajo producido por otra persona sin citarlo adecuadamente", a lo que añaden que dicha acción de copia puede hacerse tanto online como offline, es decir, a partir de material impreso o digital. Si bien la definición del término está clara, todavía existen dudas sobre qué prácticas podrían identificarse con una conducta plagiaria, ya que el plagio o ciberplagio va más allá de una copia literal o mal reformulada de las ideas de un tercero, sino que también incluye conductas como:

(i) la traducción del contenido de una fuente antes de insertarla en un documento nuevo, adjudicándose así su autoría;

(ii) la reproducción literal de una cita tras haber cerrado comillas omitiendo que las frases anteriores y/o posteriores corresponden a la misma cita;

(iii) el cambio de alguna palabra en una cita para reproducirla sin comillas;

(iv) la inclusión de datos, cifras, metodologías o procedimientos falsos o sin contrastar en un estudio para ajustar los resultados a los objetivos de la investigación;

(v) la inclusión del nombre de algunas personas en un trabajo o publicación sin haber participado en su elaboración; o

(vi) la compra y reutilización de trabajos realizados por terceros.

De ahí que nuestra aproximación a este fenómeno sea distinta cuanto mayor sea nuestro conocimiento sobre su tipología, sus implicaciones académicas y consecuencias formativas tanto para el estudiante que lo comete como para el profesorado que lo detecta.

Según nuestros profesores, de entre los casos de plagio detectados, los estudiantes han ciberplagiado en actividades prácticas evaluables (redacciones, ensayos, comentarios de texto, resúmenes de lectura), ejercicios de clase, trabajos de fin de grado y exámenes; conductas que se identificaron, no solo por intuición o experiencia docente, sino también gracias a la utilización de motores de búsqueda genéricos (Google, Yahoo, Bing, etc.) y herramientas antiplagio como Safe Assign, Turnitin o Urkund. De los casos detectados, el $32 \%$ se cometió voluntariamente, el $10 \%$ fue accidental, el $14 \%$ una mezcla de ambas opciones y el $44 \%$ restante no se identifica, bien porque corresponde al porcentaje del profesorado que no ha detectado un caso de plagio en los últimos tres años o porque se 
tiene dudas acerca de la intencionalidad con la que se cometió, una apreciación que no siempre resulta tan evidente.

En cuanto a los factores que incitan al estudiantado a cometer ciberplagio, destacan la mala gestión del tiempo de estudio, la facilidad de acceso a la información, el volumen de tareas solicitadas, la competitividad por obtener mejores resultados, la ausencia total o parcial de mecanismos de disuasión, la falta de formación en esta materia, el desconocimiento de los sistemas de citación bibliográfica, la gestión deficiente de los recursos disponibles en red y la sensación de impunidad ante un acto ilícito que no se considera grave. Motivaciones que es necesario identificar para comprender lo que induce a nuestros estudiantes a utilizar la voz ajena en un texto propio y poder actuar en consonancia.

En cuanto al enunciado sobre si el profesorado proporciona indicaciones a sus estudiantes sobre cómo elaborar un trabajo académico, el $78 \%$ contesta positivamente, el $20 \%$ no lo hace y el $2 \%$ no sabe/no contesta. Las indicaciones que comparten están relacionadas con las normas de citación de referencia (APA, ISO, MLA, etc.), la búsqueda y selección bibliográfica, el manejo de bases de datos, el uso de estrategias de reformulación de ideas y el desarrollo del análisis personal a través de la crítica de traducciones.

Por lo que respecta a la reflexión, en clase, sobre el fenómeno del ciberplagio, no todos los docentes abordan esta problemática en su asignatura. De hecho, un porcentaje elevado (42\%) no lo trata de manera sistemática -algunos porque lo consideran poco relevante o pertinente (especialmente en las asignaturas de interpretación)-, mientras que el 58\% del profesorado sí lo hace en la primera sesión de tutoría; mencionan la normativa actual de la universidad en materia de ciberplagio, les recuerdan las medidas sancionadoras en caso de detección; les explican cómo el profesorado lo detecta y qué herramientas utilizan para hacerlo; comparten ejemplos reales de casos previamente identificados para tener una visión más cercana de esta realidad e incluso les facilitan materiales (recursos, tutoriales, vídeos y enlaces ${ }^{1}$ ) para evitar caer en estas conductas. Todo ello para sensibilizar al estudiantado sobre esta problemática, evitar que sea un tema tabú en nuestro ámbito profesional y pueda atajarse como es debido.

Si bien es cierto que cada profesor lo aborda de manera distinta en el aula, desde las dos últimas ediciones, todas las guías docentes del grado incluyen un párrafo que alude

\footnotetext{
${ }^{1}$ Algunos ejemplos del material que se les facilita son:

(i) el reportaje realizado por Envoyé spécial el pasado 30 de abril de 2015, Compilatio.net https://www.youtube.com/watch? $\mathrm{v}=$ ocyscsDMc1I;

(ii) el tutorial de la University of Sydney Library, CRUE (Conferencia de Rectores de las Universidades Españolas) -REBIUN (Red de Bibliotecas Universitarias) y la Comisión Sectorial de las Tecnologías de la Información y las Comunicaciones (TIC). "El plagio y la honestidad académica" (http://www.crue.org/tutorial plagio/); y

(iii) el vídeo producido, en clave de humor, por la Universidad de Bergen, Et Plagieringseventyr. https://www.youtube.com/watch?v=Mwbw9KF-ACY.
} 
expresamente a estas conductas y a la normativa de centro $^{2}$; iniciativa que revela que los esfuerzos de nuestros docentes empiezan a estar alineados.

En cuanto a las medidas que debería tomar el profesorado para corregir y evitar estos comportamientos fraudulentos, se proponen como recurrentes las siguientes:

(i) Insistir en la importancia de citar correctamente las fuentes consultadas y respetar las ideas de los demás (formación obligatoria en la asignatura de Documentación, en la que se les enseña a gestionar, consultar, manejar y consumir información).

(ii) Facilitar al estudiantado el acceso a la normativa de citación de referencia en nuestra área.

(iii) Ofrecer formación al estudiantado sobre cómo evitar el ciberplagio (dándoles ejemplos sobre los distintos tipos de plagio, poniendo a su disposición las herramientas informáticas necesarias para que ellos mismos evalúen si han plagiado involuntariamente antes de presentar una actividad, etc.).

(iv) Informarles sobre las pautas clave para la elaboración de trabajos académicos.

(v) Proponerles actividades motivadoras que requieran reflexión y creatividad, y estén vinculadas con la realidad profesional.

(vi) Corregir las tareas con mayor profundidad.

(vii) Reducir el volumen de tareas solicitadas.

(viii) Aplicar medidas sancionadoras más severas.

(ix) Actuar, de manera coordinada, siguiendo las mismas pautas de sensibilización, concienciación y sanción que marca la normativa de centro.

(x) Mostrar tolerancia cero frente al plagio siendo intransigentes ante estas conductas.

Estas son solo algunas de las medidas que se mencionaron en la encuesta y que posteriormente se complementaron en el grupo de discusión. Al tratarse de un enunciado de respuesta abierta, únicamente hemos relacionado las diez primeras propuestas, aquellas que evidencian un mayor grado de acuerdo por parte del profesorado, si bien el listado es más extenso. Su sistematización y análisis, así como las iniciativas planteadas para su consecución surgidas durante la sesión serán objeto de un estudio posterior.

También es importante comentar las medidas que, según el profesorado, el centro debería implementar para aumentar la sensibilización hacia este fenómeno. Entre las más interesantes destacan (i) la formación sobre ciberplagio, impartida por expertos y dirigida a profesores, estudiantes y responsables de centro; (ii) la constitución de una comisión de ética a la que tanto profesores como estudiantes pudieran dirigirse para plantear sus dudas; (iii) el

2 "Dado que la Universidad Internacional de Valencia (VIU) en su Reglamento de régimen disciplinario de los estudiantes (http:/www.viu.es/normativa/) incluye, entre sus faltas muy graves, el plagio total o parcial de obras intelectuales de cualquier tipo, el estudiante deberá conocer y reglamento y responsabilizarse de que todas sus producciones (orales o escritas) sean redactadas de forma creativa y personal. En el caso de que el profesor detecte copia o plagio literal de cualquier fuente, la nota obtenida en la tarea asignada será 0" (párrafo incluído en todas las guías docentes del Grado en Traducción e Interpretación de la Universidad Internacional de Valencia). 
establecimiento de un protocolo de actuación antiplagio (no solo desde una perspectiva sancionadora, sino de concienciación); (iv) la puesta en marcha de campañas de sensibilización ante estas prácticas ("un plagio, un cero", por ejemplo); y (v) la posibilidad de proporcionar incentivos académicos por buenas prácticas (para ambos colectivos), lo que podría ayudar a reducirlas y evitarlas.

Todas estas medidas se trataron de nuevo en el grupo de discusión en el que se aprovechó para valorar su aplicación en nuestro grado. Sin duda, estas propuestas requieren de procedimientos distintos de ejecución (tanto a nivel de recursos como de tiempo), pero lo interesante es que se han podido identificar como necesarias en el seno de nuestra institución gracias a este estudio. Es necesario, pues, seguir aunando esfuerzos para remar todos en la misma dirección. No se trata de jugar al ratón y al gato, o de colgarse medallas cada vez que se identifica un caso de plagio, sino de motivar al estudiante a pensar por sí mismo y enseñarle el valor de la integridad y el respecto hacia aquello que no le pertenece, aunque las redes le faciliten el acceso.

Por otro lado, cabe incidir en la importancia el corpus informativo que recabamos a partir del grupo de discusión, en el que se abordaron las cuestiones anteriormente indicadas.

Durante la sesión programada, se leyeron algunas definiciones del término ciberplagio utilizadas por expertos en la materia y se compartieron los resultados más relevantes de la encuesta, previamente cumplimentada por el profesorado. Las preguntas que se les plantearon giraron en torno a tres ejes temáticos: (i) la responsabilidad a asumir por el profesorado en caso de detección de estas conductas; (ii) el procedimiento a seguir tras su detección; y (iii) las buenas prácticas a adoptar por parte de la comunidad universitaria y, en especial, el centro y su profesorado.

El profesorado coincidió en su papel clave en la lucha contra este fenómeno, puesto que son ellos quienes detectan estas conductas deshonestas y deben comunicarlas al alumnado. El claustro se mostró en todo momento implicado en este tema, pero con la puntualización de que era imprescindible el apoyo de la institución académica para combatir esta práctica. Al parecer, no todos los centros de educación superior adoptan una postura firme ante esta problemática. Algunos profesores afirmaron que, en otras universidades en las que habían colaborado, el plagio/ciberplagio seguía siendo un tema tabú al que no se le confería la importancia que merece. La institución debería, pues, adoptar la responsabilidad que le corresponde como empleadora de su profesorado y ofrecer los recursos formativos necesarios para controlar y disuadir estas prácticas.

Por otra parte, los docentes afirmaron desconocer la existencia de un protocolo de actuación de referencia mediante el cual reportar a la coordinación o dirección de título un caso de plagio. En este sentido, se acordó crear un registro de conductas deshonestas que permitiera al profesorado recabar esta información, sistematizarla y compartirla en posteriores reuniones de claustro. Dicho registro permitiría materializar las distintas experiencias identificadas a lo largo de cada curso académico parallegar a conclusiones y plantear acciones futuras de manera coordinada. También se sugirió la posibilidad de que la universidad contara con un grupo de profesores externos, especialistas en la materia, que determinaran en última instancia la veracidad de los casos de ciberplagio detectados. 
Finalmente, y entre las estrategias y buenas prácticas compartidas, el profesorado consideró que la institución debería realizar un importante trabajo de sensibilización a todos los niveles y un esfuerzo por implicar a todos los colectivos en la lucha contra el robo de ideas sistemático; la comunidad universitaria requiere, pues, sentir esa afirmación pública por parte del centro respecto a su postura ante esta problemática de gran calado (a través de campañas publicitarias, formación continua, protocolos de actuación de acceso público, etc.). El respaldo institucional es, sin duda, clave para la puesta en marcha de gran parte de las iniciativas comentadas; sin él, el fenómeno del ciberplagio seguirá creciendo y cangrenando el sistema educativo universitario.

\section{Conclusiones}

Ambas herramientas de medición nos han permitido llegar a la conclusión de que el ciberplagio es un problema que debemos atajar mediante distintas acciones y con el compromiso de todas las partes implicadas. La opinión de los profesores es clara y su implicación, determinante, pero no suficiente si el centro no les apoya en las actuaciones que lleven a cabo en sus materias y grupos, y si no existe una postura institucional firme que sea conocida por todos (profesores y alumnos).

A tenor de los resultados obtenidos, no cabe duda de que estos serán de gran utilidad para seguir trabajando en la percepción del ciberplagio por parte del colectivo que se enfrenta a él en primera línea de batalla. Tanto la encuesta como el grupo de discusión son susceptibles de ser ampliados y pueden nutrirse de aspectos más concretos que enriquezcan estos resultados.

Consideramos que este estudio no está exento de limitaciones. Sin duda, el reto será ampliar la muestra de profesores participantes para seguir reflexionando sobre este fenómeno. Por la naturaleza del entorno de aprendizaje en el cual se basa el estudio y el perfil de su profesorado, no siempre resulta fácil. En cualquier caso, los resultados se analizaran y cruzarán con la información recopilada en las encuestas dirigidas a estudiantes, permitiéndonos así tener una visión mucho más amplia sobre este problema.

\section{Referencias}

Arias PoU, M. (2012). "La gestión de la propiedad intelectual en la Universidad" en Diario La Ley, 7980, Sección Doctrina, 10 de diciembre de 2012 (ref. D-434). Madrid: La Ley.

Bautista Buenfil, F., SÁnchez Escobedo, P. A., Canto Herrera, P. J. (2017). "Plagio en los posgrados de ciencias sociales en una universidad estatal de México" en Educación y Ciencia, vol. 6, núm. 47, pp. 82-97.

COMAS, R., SuREDA, J., PASTOR, M. y MOREY, M. (2011). "La búsqueda de información con fines académicos entre el alumnado universitario" en Revista Española de Documentación

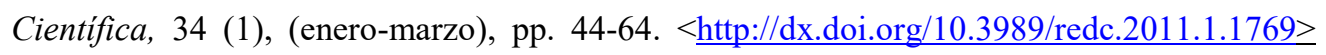
[Consulta: 26 de mayo de 2019] 
Cueva Lobelle, A. y OCHOA SIERRA, L. (2015). "Representaciones sociales de profesores y estudiantes acerca del plagio: estudio comparativo" en Revista de Investigaciones UCM, 15 (25), pp. 60-69.

DICKEY, M. D. (2004). "The impact of web-logs (blogs) on student perceptions of isolation and alienation in a web-based distance-learning environment” en Open Learning, vol. 19 (3), pp. 279-291.

Domínguez PÉReZ, D. A. (2015). "La tecnodependencia, una consecuencia del plagio electrónico de los trabajos académicos: el caso de una universidad privada" en Revista Multidisciplinaria de Avances de Investigación, 2 (2), mayo-agosto, pp. 14-29.

FRANCE 2 (2015). “Compilatio.net”. Reportaje realizado por Envoyé spécial emitido el 30 de abril de 2015. $<$ https://www.youtube.com/watch? $\mathrm{v}=$ ocyscsDMc1I $>$ [Consulta: 26 de mayo de 2019]

Gallent TorRes, C. y Tello Fons, I. (2017). "Percepción del alumnado de traducción de la Universidad Internacional de Valencia (VIU) sobre el ciberplagio académico" en Revista Digital de Investigación en Docencia Universitaria, 11 (2), pp. 90-117. $<$ http://dx.doi.org/10.19083/ridu.11.563 > [Consulta: 26 de mayo de 2019]

GALlent ToRRes, C. y Tello Fons, I. (2018). "Le cyberplagiat dans le cadre de l'éducation supérieure espagnole: explorer les causes pour atteindre des solutions concrètes" en $\begin{array}{llll}\text { Synergies } \quad \text { Espagne, } & 11, & \text { 195-212. }\end{array}$ $<$ https://www.gerflint.fr/Base/Espagne11/gallent tello.pdf> [Consulta: 26 de mayo de 2019]

JONES, I. M. (2007). "Cyber-plagiarism: Different method-same song" en Proceedings of the Academy of Educational Leadership, vol. 12, núm. 2, pp. 17-21.

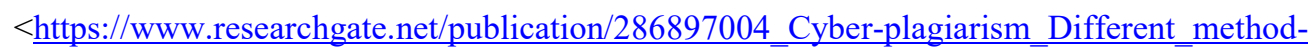
same song > [Consulta: 26 de mayo de 2019]

Medina díAZ, M. DEL R. y Verdejo CARRión, A. L. (2016). "Una mirada a la deshonestidad académica y el plagio estudiantil en algunas universidades de siete países de América Latina", pp.

$1-16$. $<$ http://reposital.cuaed.unam.mx:8080/jspui/bitstream/123456789/4673/1/VE16.779.pdf $>$ [Consulta: 26 de mayo de 2019]

PARK, C. (2003). "In Other (People's) Words: Plagiarism by university students-literature and lessons" en Assessment and Evaluation in Higher Education, 28 (5), pp. 471-488.

PARDO IRANZO, V. (2014). "La docencia online: ventajas, inconvenientes y forma de organizarla" en Revista Boliviana de Derecho, 18, pp. 622-635.

PAVlakis, A. Y KaITElidou, D. (2012). "Burnout syndrome in students of a distance learning program: The Open University of Cyprus experience" en European Journal of Open, Distance and E-Learning, 2012/1. $<$ http://www.eurodl.org/materials/contrib/2012/Pavlakis_Kaitelidou.pdf $>$ [Consulta: 26 de mayo de 2019] 
Ronda-Pérez, E., Seguí-Crespo, M., Tauste Francés, A., Martínez Martínez, J. M., y Esteve-FAuBEL, J. M. (2015). "Experiencia piloto de evaluación de una intervención para evitar el plagio académico en alumnos universitarios" en Investigación y propuestas innovadoras de redes UA para la mejora docente [recurso electrónico]. Alicante: Universidad de Alicante, Vicerrectorado de Estudios, Formación y Calidad, Instituto de Ciencias de la Educación (ICE), pp. 1932-1947.

SARLAUSKIENEA, L. Y STABINGIS, L. (2014). "Understanding of plagiarism by the students in HEIs of Lithuania" en Procedia - Social and Behavioral Sciences, 110 (2014), pp. 638 - 646.

THE UnIVERSITY OF SIDNEY LIBRARY, CONFERENCIA DE RECTORES DE LAS UNIVERSIDADES ESPaÑolas (CRUE), RED de bibliotecas Universitarias (REBIUN) Y LA COMisión SECTORIAL de las TeCNOLOGÍAS DE LA INFORMACIÓN Y LAS COMUNICACIONES (TIC) (n.d).

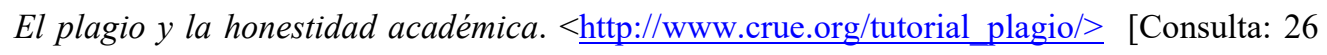
de mayo de 2019]

UNDERWOOD, J. Y SZABO, A. (2003). "Academic offences and e-learning: individual propensities in cheating" en British Journal of Educational Technology, vol. 34, n. ${ }^{\circ}$ 4, pp. 467-477.

UNIVERSIDAD INTERNACIONAL DE VALENCIA (VIU) (2019). Reglamento de régimen

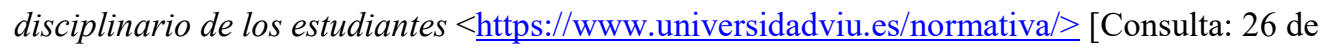
mayo de 2019]

UNIVERSITY OF BERGEN UIB (2010). "Et Plagieringseventyr $<$ https://www.youtube.com/watch?v=Mwbw9KF-ACY $>$ [Consulta: 26 de mayo de 2019] 